\title{
Asymmetric Gap J unctional Coupling Between Glial Cells in the Rat Retina
}

\author{
KATHLEEN R. ZAHS* AND ERIC A. NEWMAN \\ Department of Physiol ogy, University of Minnesota, School of Medicine, \\ Minneapolis, Minnesota 55455
}

KEY WORDS $\begin{aligned} & \text { astrocyte; Müller cell; gap junction; dye coupling; glia; intercellular } \\ & \text { coupling }\end{aligned}$

ABSTRACT Gap junctional communication between glial cells is thought to play a role in $\mathrm{K}^{+}$spatial buffering, in the propagation of inter-astrocytic $\mathrm{Ca}^{2+}$ waves, and in glial-neuronal signaling. In the present study, we characterize dye coupling between astrocytes, and between astrocytes and Müller cells, in the isolated rat retina.

Whole-cell patch recordings were obtained from retinal astrocytes and Müller cells and the cells filled with Lucifer Yellow and neurobiotin. Spread of Lucifer Yellow to two to ten neighboring astrocytes occurred in $90 \%$ of the astrocyte recordings. After fixation and incubation of the retina with fluorescent conjugated streptavidin, neurobiotin was seen to label clusters of 13-88 astrocytes, as well as >100 Müller cells. In contrast, when Müller cells were filled with Lucifer Yellow and neurobiotin, both tracers were confined solely to the recorded Müller cell. The uncoupling agents octanol, halothane, and doxyl-stearic acid were tested for their ability to uncouple retinal glia in situ. All three agents eliminated the visible spread of Lucifer Yellow from the injected astrocyte and the spread of neurobiotin into Müller cells. However, only doxyl-stearic acid combined with octanol eliminated the spread of neurobiotin between astrocytes.

These results demonstrate that astrocytes in the rat retina are coupled to each other and to Müller cells. The astrocyte-to-Müller cell coupling is asymmetric, allowing transfer of the tracer in the forward direction only. In addition, astrocyte-to-Müller cell coupling is more sensitive to the uncoupling agents tested than is astrocyte-to-astrocyte coupling. GLIA 20:10-22, 1997. @ 1997 Wiley-Liss, Inc.

\section{INTRODUCTION}

Intercellular coupling between astrocytes is common in vivo (Binmoller and Müller, 1992; Massa and Mugnaini, 1985) and in vitro (Enkvist and McCarthy, 1992, 1994; Massa and Mugnaini, 1982; Ransom and Kettenmann, 1990). Astrocytes are al so coupled to other types of glial cells, including oligodendrocytes (Massa and Mugnaini, 1982, 1985; Ransom and Kettenmann, 1990; Venance et al., 1995) and Müller cells (Robinson et al., 1993). Astrocyte coupling is believed to have an important role in the regulation of extracellular $\mathrm{K}^{+}$: $\mathrm{K}^{+}$ spatial buffer currents flowing through the astrocyte syncytium prevent the local accumulation of extracelluIar $\mathrm{K}^{+}$in areas of high neuronal activity (Orkand et al., 1966). J unctional coupling also mediates the propagation of $\mathrm{Ca}^{2+}$ waves between astrocytes (Enkvist and McCarthy, 1992; Finkbeiner, 1992; Nedergaard, 1994).
In addition, the absence of gap junctions is correlated with proliferative activity in someglial tumors (Charles et al., 1992; Naus et al., 1993; Tani et al., 1973; Zhu et al., 1991).

The mammalian retina is a particularly good model system for studying the regulation and functions of glial cell coupling in situ. Astrocyte processes ramify mainly in two dimensions at the vitreal surface of the retina. These cells are readily identifiable in isolated retinas and are accessible for el ectrophysiological recording. It has recently been reported that, in therabbit

\footnotetext{
Received 8 August 1996; accepted 10 December 1996
}

*Correspondence to: Dr. Kathleen R. Zahs, University of Minnesota, School of Medicine, Department of Physiology, Millard 6-255, 435 Delaware Street S.E. Minneapolis, MN 55455.

Contract grant sponsor: National Institutes of Health; Contract grant number: EY10383. 
TABLE 1. Membraneproperties of retinal gliaa

\begin{tabular}{lrrrrr}
\hline & Control & Octanol & Halothane & DSA & DSA/octanol \\
\hline Astrocytes & & & & & \\
$\mathrm{V}_{\mathrm{m}}(\mathrm{mV})$ & $-85 \pm 1(43)$ & $-86 \pm 1(66)$ & $-85 \pm 1(10)$ & $-86 \pm 1(14)$ & $-84 \pm 1(11)$ \\
$\mathrm{R}_{\mathrm{i}}(\mathrm{M} \Omega)$ & $29 \pm 2(43)$ & $27 \pm 2(66)$ & $31 \pm 3(10)$ & $53 \pm 11(14)^{* *}$ & $71 \pm 15(11) * * *$ \\
Müller cells & $-93 \pm 1(25)$ & $-92 \pm 1(15)$ & $-90 \pm 1(11)$ & N.A. & $-88 \pm 2(6)^{*}$ \\
$\mathrm{~V}_{\mathrm{m}}(\mathrm{mV})$ & $24 \pm 2(25)$ & $26 \pm 3(15)$ & $26 \pm 4(11)$ & N.A. & $38 \pm 13(6)$ \\
$\mathrm{R}_{\mathrm{i}}(\mathrm{M} \Omega)$ & & &
\end{tabular}

aMembrane potentials $\left(\mathrm{V}_{\mathrm{m}}\right)$ and input resistances $\left(\mathrm{R}_{\mathrm{i}}\right)$ of glial cells recorded in the whole-cell configuration in isolated rat retinas perfused with control-Ringer's solution, Ringer's with $500 \mu \mathrm{M}$ octanol, Ringer's bubbled with $\mathrm{O}_{2}$ with $4 \%$ halothane, Ringer's with $50-100 \mu \mathrm{M}$ DSA, and Ringer's with $100 \mu \mathrm{M}$ DSA plus $500 \mu \mathrm{M}$ octanol. Values are mean \pm SEM. Numbers in parentheses show the number of cells included in each sample. *, P $<0.05$; **, P $<0.001$; ***, P $<10^{-4}$, compared with control, Student's t-test.

retina, astrocytes are coupled to Müller cells, the principal glial cell of the retina, in addition to being coupled to each other (Robinson et al., 1993). This coupling was most prominently demonstrated when biocytin was used as the tracer, although the spread of the larger Lucifer Yellow was sometimes observed. Especially intriguing was the finding that the spread of tracer is unidirectional, occurring only in the direction of astrocyte to Müller cell.

In the current study, we have investigated the pattern of dye coupling among glial cells of the isolated rat retina, using Lucifer Yellow and a biotin derivative, neurobiotin, as tracers. Our findings are consistent with those reported in the rabbit, showing that: 1) both Lucifer Yellow and neurobiotin introduced into a single astrocyte spread to neighboring astrocytes; 2 ) neurobiotin spreads to Müller cells; and 3) the spread of neurobiotin to Müller cells is unidirectional. I n contrast to the earlier study, Lucifer Yellow was never observed to spread from astrocytes to Müller cells. In addition, we find that the coupling between astrocytes and Müller cells is much more sensitive to the effects of uncoupling agents than is the coupling between astrocytes. Concentrations of uncoupling agents that prevented the visible spread of Lucifer Yellow did not abolish the spread of neurobiotin between astrocytes. This result demonstrates that observing the spread of Lucifer Yellow can be misleading as an indicator of whether or not cells are coupled.

\section{MATERIALS AND METHODS Preparation of I solated Retinas}

Male Long-Evans rats (150-400 g) were deeply anesthetized with sodium pentobarbital $(200 \mathrm{mg} / \mathrm{kg})$, administered intraperitoneally, and perfused transcardially with approximately $50 \mathrm{ml}$ of Ringer's solution, after clamping the descending aorta. The eyes were removed and the retinas dissected into oxygenated Ringer's solution. Most of the vitreous was teased away using fine forceps. Retinas were stored at room temperature in oxygenated Ringer's solution. Prior to electrophysiological recording, pieces of retina were incubated for $20 \mathrm{~min}$ at room temperature in a mixture of collagenasedispase $(2 \mathrm{mg} / \mathrm{ml})$, DNAase $(0.1 \mathrm{mg} / \mathrm{ml})$, and the fluorescent nuclear stain bisbenzimide $(10 \mu \mathrm{M}$, H oechst 33342$)$. Following enzyme incubation, the retinal surface was gently rinsed with a stream of Ringer's solution. The bisbenzimide stained the cell nuclei so astrocyte somata could be made visible, and the enzyme treatment allowed patch el ectrodes to form high-resistance seals, probably by removing the basal lamina and any remaining vitreous.

\section{Whole-Cell Recording}

The enzymetreated retina was placed, vitreal side up, onto a piece of polycarbonate filter membrane, with a window cut in the center to permit brightfield illumination of the tissue. The tissue was affixed to the filter membrane using a device that applied suction to the edges of the tissue, but not to the central portion over the window. The retina and filter membrane were then mounted in a perfusion chamber and held in place with nylon mesh. The retina was imaged with an upright microscope equipped with epifluorescence, a cooled CCD camera (I magePoint, Photometrics, Tucson, AZ), video monitor, and super-VHS recorder. The tissue was superfused at room temperature (approximately $22^{\circ} \mathrm{C}$ ) with Ringer's solution, at $\sim 10 \mathrm{ml} / \mathrm{min}$, which exchanged the volume in the chamber approximately once every $3 \mathrm{~s}$.

The retinal surface was viewed with oblique brightfield illumination, achieved by decentering the brightfield condenser. The tissue was bri efly illuminated with ultraviolet light to locate astrocytes for whole-cell recording, and the positions of bisbenzimide-stained nuclei noted on the video monitor. The patch pi pette was then positioned over one of these nuclei, and gentle suction applied to the back of the pipette until a $\mathrm{G} \Omega$ seal was formed. The membrane was then ruptured to establish the whole-cell recording. Membrane potential was recorded using an Axopatch-1D amplifier (Axon Instruments, Foster City, CA). Input resistance was monitored with depolarizing current pulses.

Whole-cell recordings were maintained for $5 \mathrm{~min}$, at which point the tissue was illuminated for Lucifer Yell ow fluorescence, and a video image of the live tissue was recorded. The tissue and membrane support were then immediately removed from the perfusion chamber and fixed in $4 \%$ paraformaldehyde, $0.1 \%$ glutaraldehyde, and $0.2 \%$ picric acid in $0.15 \mathrm{M}$ phosphate buffer, $\mathrm{pH}$ 7.4, for $25 \mathrm{~min}$ at room temperature. Less than a minute elapsed between the time the whole-cell record- 

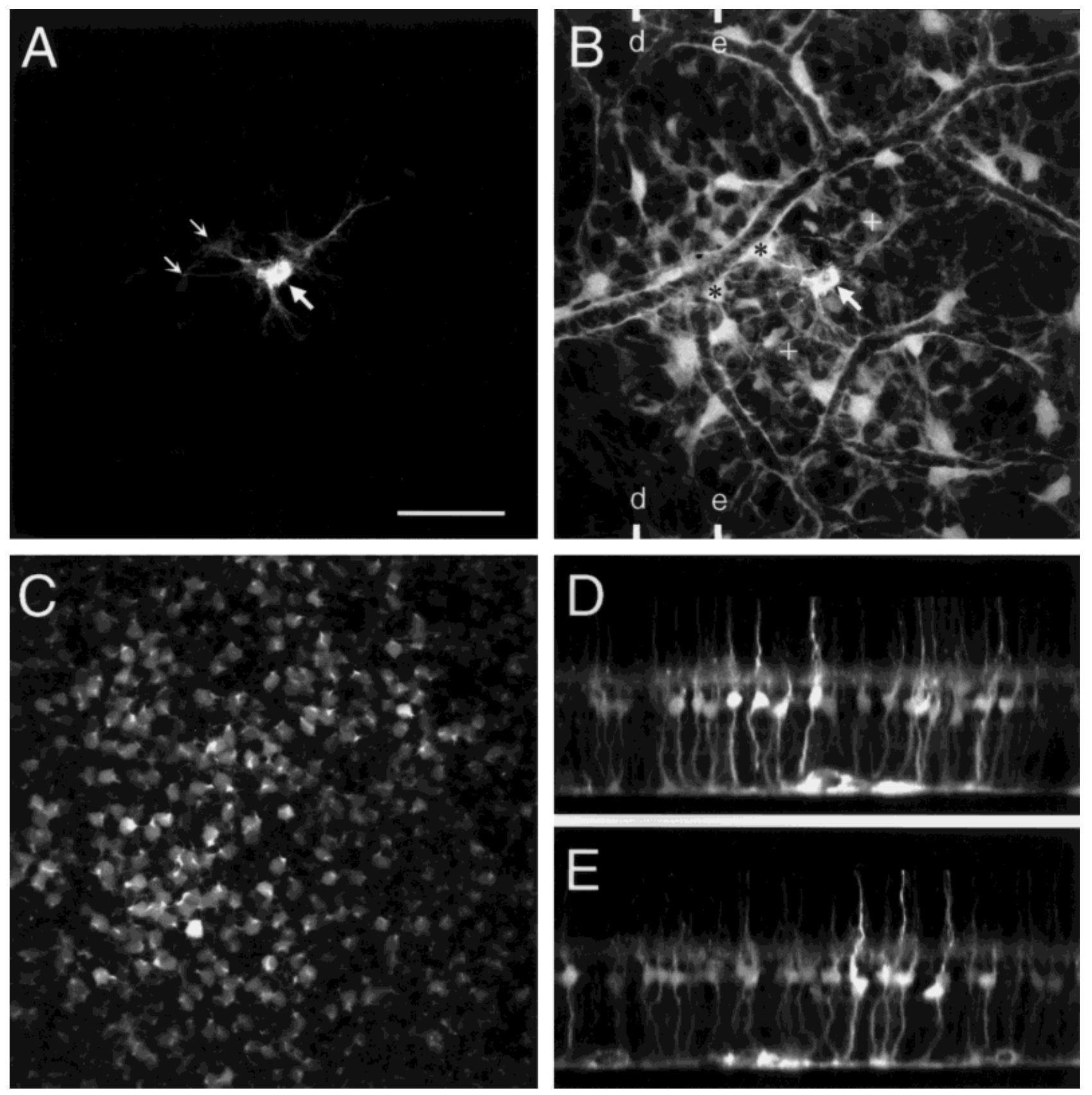

Fig. 1. Tracer coupling between glial cells of an isolated retina perfused with control Ringer's solution. A single astrocyte was filled with Lucifer Yellow and neurobiotin from a patch pipette. A: Lucifer yellow labeling. Superposition of confocal images through $10 \mu \mathrm{m}$ at the vitreal surface of the retina. The large arrow indicates the recorded astrocyte, and small arrows indicate two coupled astrocytes, with Lucifer Yellow still visible after fixation and processing. B: Neurobiotin labeling. Superposition of confocal images through the same volume shown in A. The recorded cell is again indicated by the arrow. Neurobiotin spread from the recorded cell to astrocytes and Müller cells throughout the field of view. Asterisks, neurobiotin labeling of the

ing was discontinued and the time the tissue was placed in fixative.

Following fixation, the tissue was briefly rinsed three times in Dulbecco's phosphate-buffered saline (D-PBS, $\mathrm{pH}$ 7.4) with $1 \%$ Triton-X100 and then rinsed overnight

same astrocyte somata indicated by the small arrows in A; crosses, neurobiotin-labeled Müller cell endfeet. C: Neurobiotin labeling. Superposition of confocal images through a 15- $\mu$ m-deep slab of retina, within the inner nuclear layer. The bright profiles are labeled Müller cell somata filled following injection of the single astrocyte shown in A. D and E: Neurobiotin labeling. Müller cells are filled throughout their length following injection of the single astrocyte shown in $A$. The two images are three-dimensional reconstructions of the retina in a plane orthogonal to the surface. Two 10- $\mu$ m-thick slices are shown, taken at the levels indicated in B; photoreceptor side is up, and vitreal side is down. Scale bar in $A=50 \mu \mathrm{m}$ and applies to all panels.

in $\mathrm{D}-\mathrm{PBS} / 1 \%$ Triton at $4^{\circ} \mathrm{C}$. The retina was then incubated for 2 days with streptavidin conjugated to Cy3 $(20 \mu \mathrm{g} / \mathrm{ml})$ or to Cy5 $(29 \mu \mathrm{g} / \mathrm{ml})$, rinsed overnight in D-PBS, mounted on a microscope slide, and coverslipped with Vectashield mounting medium (Vector 

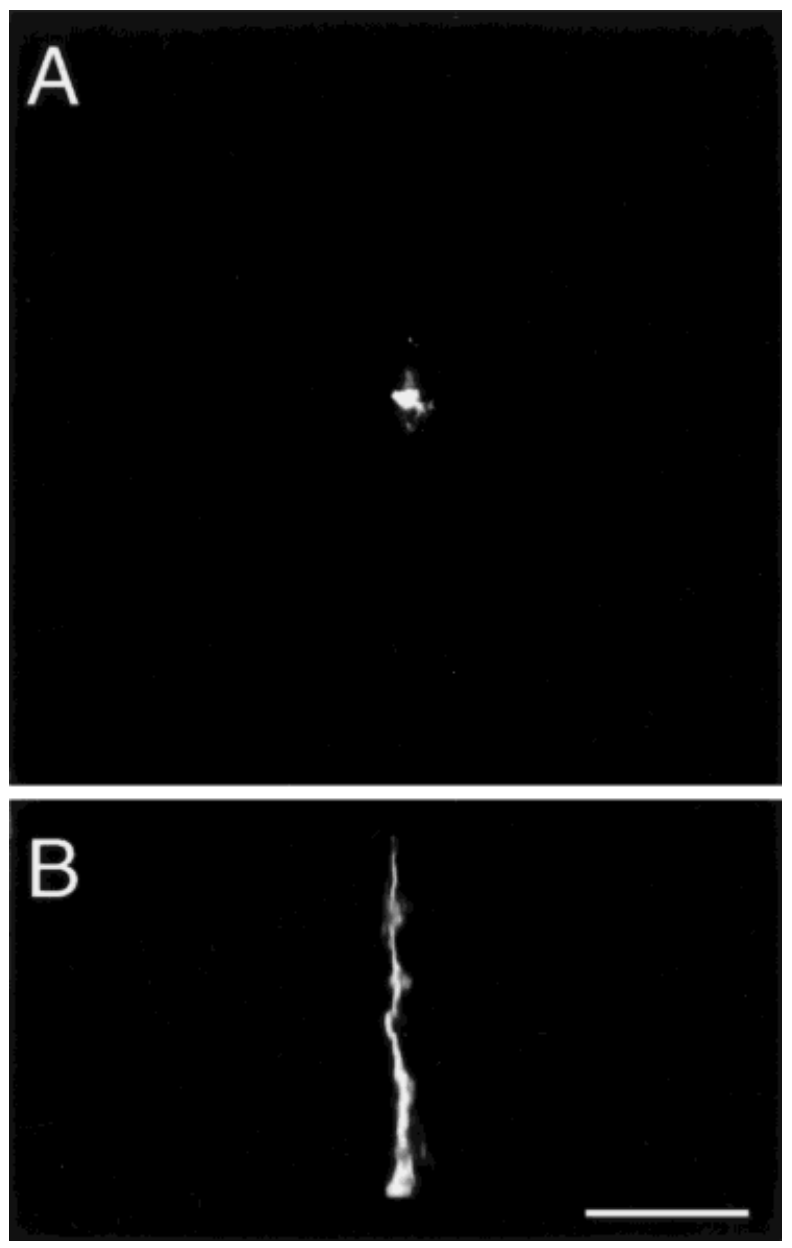

Fig. 2. Tracer coupling between astrocytes and Müller cells is unidirectional. A single Müller cell was recorded in the whole-cell configuration and filled with neurobiotin. The tracer did not spread from this Müller cell to astrocytes or to other Müller cells. A: View of the retinal surface. The endfoot of the single, labeled cell is visible. B: Longitudinal view of the labeled Müller cell from A, after computer reconstruction of 100 optical sections. Again, label is visible only in the recorded cell. Scale bar $=50 \mu \mathrm{m}$.

Laboratories, Burlingame, CA) to retard fading of the fluorescent tag.

\section{Confocal Microscopy and I mage Reconstruction}

After processing with the streptavidin-conjugated fluorophore, retinas were viewed with conventional fluorescence microscopy and confocal mi croscopy (Leica TCS4D or Zeiss LSM 10 laser scanning microscope). To view Lucifer Yellow-labeled astrocytes at the vitreal surface, 15-20 optical sections were taken at $1 \mu \mathrm{m}$ intervals parallel to the retinal surface $(40 \times$ oil immersion objective, $488 \mathrm{~nm}$ excitation, $530 \mathrm{~nm}$ bandpass emission filter). To view neurobiotin-filled astrocytes and Müller cells, 100 optical sections were taken at 1 $\mu \mathrm{m}$ intervals through the depth of the retina (40× oil immersion objective; $543 \mathrm{~nm}$ excitation with a $590 \mathrm{~nm}$ long pass emission filter for Cy3; $633 \mathrm{~nm}$ excitation with a $665 \mathrm{~nm}$ emission filter for Cy5).

I mages of the labeled tissue were reconstructed using a Silicon Graphics I nstruments computer running VoxelView (Vital Images, Fairfield, IA) software. Optical sections taken through a depth of $10 \mu \mathrm{m}$ at the vitreal surface of the retina were superimposed to produce images of astrocytes and Müller-cell endfeet. I mages of Müller cell somata were generated by the superposition of 15 optical sections taken within the inner nuclear layer. To view labeled Müller cells throughout their length, we generated computer reconstructions of 10$\mu \mathrm{m}$-thick slices in a plane orthogonal to the retinal surface.

\section{Immunocytochemical Detection of L ucifer Yellow}

In a few experiments in octanol-treated retinas, Lucifer Yellow (9 $\mathrm{mM})$ but not neurobiotin was included in the patch pipette. Whole-cell recording was performed as described above. Immediately after removal of the patch pipette, the retina was fixed for $3 \mathrm{~h}$ at $4^{\circ} \mathrm{C}$ in $3.5 \%$ paraformal dehyde, $0.1 \%$ glutaraldehyde in 0.1 $\mathrm{M}$ phosphate buffer (PB), pH 7.4, and then rinsed for 6 $\times 10 \mathrm{~min}$ in $0.1 \mathrm{M} \mathrm{PB}$. Endogenous peroxidases were inhibited by incubation for $1 \mathrm{~h}$ at room temperature in $0.5 \% \mathrm{H}_{2} \mathrm{O}_{2}$ in $0.1 \mathrm{M}$ Tris buffer (TB), followed by rinsing for $2 \times 10 \mathrm{~min}$ in $0.1 \mathrm{M}$ TB and then $3 \times 10 \mathrm{~min}$ in $0.1 \mathrm{M}$ Tris-buffered saline (TBS). Retinas were then incubated for $2 \mathrm{~h}$ at room temperature in 3\% normal goat serum (NGS) $+3 \%$ bovine serum al bumin (BSA) $+1 \%$ Triton-X100 in $0.05 \mathrm{M}$ TBS, rinsed for $6 \times 10 \mathrm{~min}$ in 0.1 $M$ TBS, incubated for 3 days at $4^{\circ} \mathrm{C}$ in anti-Lucifer Yellow (1:500 in $0.05 \mathrm{M}$ TBS with $1 \% \mathrm{NGS}+0.2 \%$ BSA + 1\% Triton-X100), rinsed for $6 \times 20 \mathrm{~min}$ in $0.1 \mathrm{M}$ TBS, incubated for $3 \mathrm{~h}$ at room temperature in goat-antirabbit antibody conjugated to horseradish peroxidase (HRP) (1:500 in the same diluent used for primary antibody), rinsed for $2 \times 10 \mathrm{~min}$ in $0.1 \mathrm{M}$ TBS followed by $3 \times 10$ min in $0.1 \mathrm{M} \mathrm{TB}, \mathrm{pH} 7.6$, and reacted using $\mathrm{DAB}$ as the chromagen. Retinas were viewed with an Olympus BH-2 microscope with a $40 \times$ oil immersion objective.

\section{Solutions}

Control Ringer's solution was composed of (in $\mathrm{mM}$ ): $\mathrm{KCl} 2.5, \mathrm{NaCl} 140, \mathrm{CaCl}_{2} 3, \mathrm{MgCl}_{2}$ 0.5, $\mathrm{N}$-[2-hydroxyethyl ]piperazine-N'-[2-ethanesulfonic acid] (HEPES) 5, and glucose 15; the pH was adjusted to 7.4 with $\mathrm{NaOH}$. The solution was continuously bubbled with oxygen. In some experiments, an uncoupling agent was added to the perfusate. 1-Octanol was added directly to the control-Ringer's solution, while hal othane was introduced by adding it to the $\mathrm{O}_{2}$ gas with a halothane vaporizer. A third uncoupling agent, 16-doxyl-stearic 

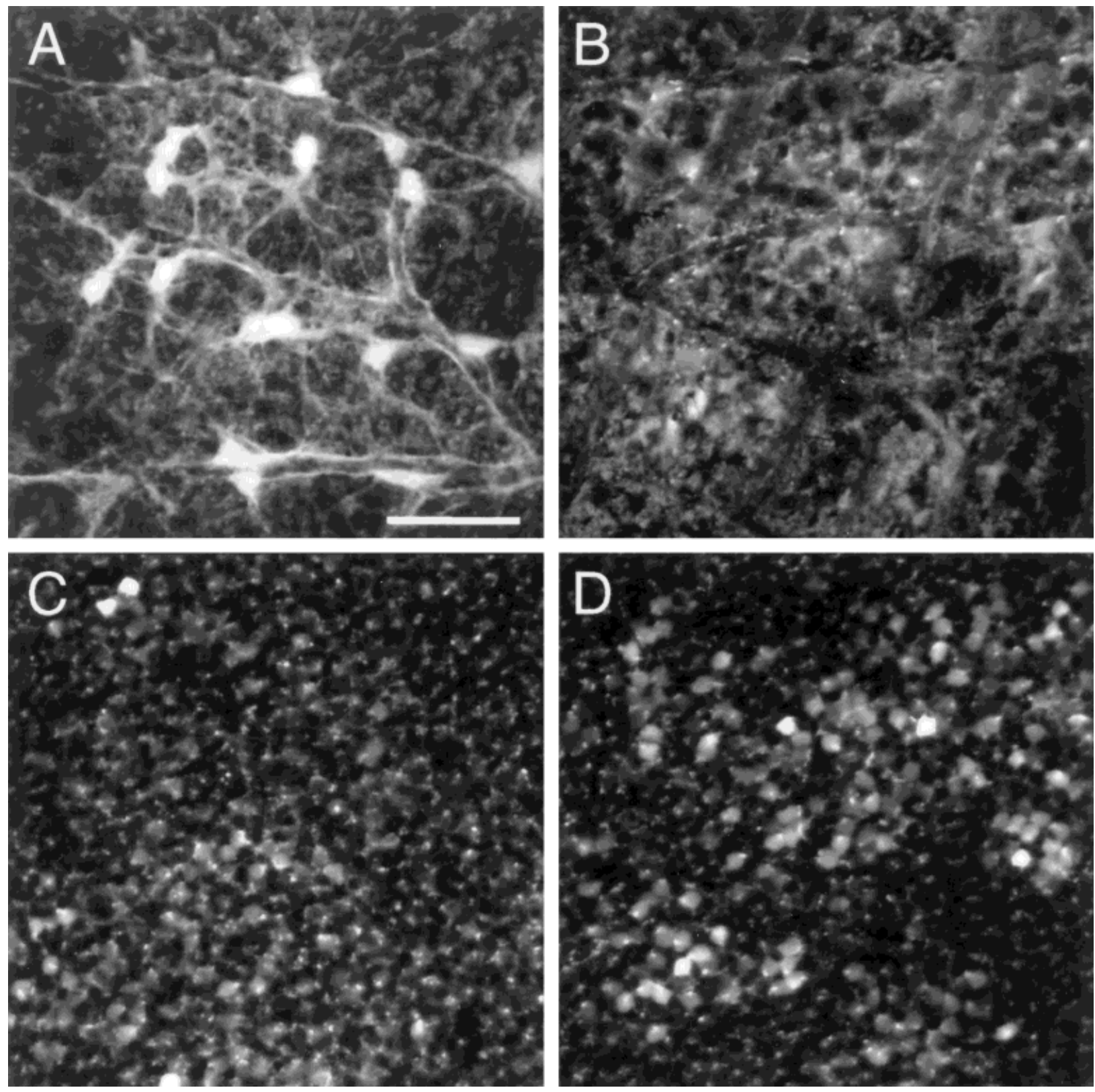

Fig. 3. Neurobiotin can diffuse between astrocytes, but is trapped in Müller cells. A: Surface view of the retina. A single astrocyte was filled with neurobiotin from a patch pipette, and the tissue was fixed immediately after removal of the pipette. A cluster of astrocytes, labeled after spread of neurobiotin from the injected cell, is visible, as are the endfeet of Müller cells. B: Surface view of a different region of the retina shown in A. A single astrocyte in the middle of this field was filled with neurobiotin from a patch pipette, but the tissue was not

acid (DSA), was largely insoluble in the Ringer's solution, so DSA was prepared as a $50 \mathrm{mM}$ stock solution in ethanol. Working solutions were prepared immediately before use by adding the stock solution to pre-oxygenated Ringer's solution in a sonicator.

The patch pipette solution contained (in $\mathrm{mM}$ ): $\mathrm{NaCl}$ $5, \mathrm{KCl} 120, \mathrm{CaCl}_{2} 1, \mathrm{MgCl}_{2}$ 7, ethylene glycol-bis-(baminoethyl ether) $\mathrm{N}, \mathrm{N}, \mathrm{N}^{\prime}, \mathrm{N}^{\prime}$-tetraacetic acid (EGTA) 5,

fixed until $20 \mathrm{~min}$ after the removal of the patch pipette. During this time, neurobiotin could continue to diffuse between astrocytes, but it could not diffuse out of the Müller cells. Only the endfeet of labeled Müller cells are visible, the neurobiotin concentration having reached undetectable levels in the astrocytes. $\mathbf{C}$ and $\mathbf{D}$ : Reconstructions of the inner nuclear layer below the regions of retina shown in $A$ and $B$ respectively, showing the somata of labeled Müller cells. Scale bar = $50 \mu \mathrm{m}$ and applies to all panels.

$\mathrm{Na}_{2}$ ATP 5, HEPES 5, Lucifer Yellow $\mathrm{CH} 2$ or 9, and neurobiotin 11; the $\mathrm{pH}$ was adjusted to 7.2 with $\mathrm{KOH}$.

HEPES, EGTA, $\mathrm{Na}_{2} \mathrm{ATP}$, 1-octanol, DSA, Lucifer Yellow, DNAase, DAB, and bisbenzimide were obtained from Sigma (St. Louis, MO); neurobiotin and antiLucifer Yellow from Molecular Probes (Eugene, OR); collagenase-dispase and HRP-goat-anti-rabbit antibody from Boehringer-Mannheim (Indianapolis, IN); 
and streptavidin conjugated to Cy3 or Cy5 from J ackson I mmunoResearch Laboratories (West Grove, PA).

\section{RESULTS}

\section{Patterns of Interglial Coupling Revealed by Lucifer Yellow and Neurobiotin}

\section{Lucifer yellow spreads to neighboring astrocytes and neurobiotin spreads to astrocytes and Müller cells}

Retinal glial cells were recorded with patch electrodes in the whole-cell configuration and filled with Lucifer Yellow (2 or $9 \mathrm{mM}$ ) and neurobiotin (11 mM), contained in the pipette solution. Recordings were obtained from 43 astrocytes in retinas perfused with control Ringer's sol ution. Astrocytes were recognized by their location at the vitreal surface of the retina, by their morphology as revealed by filling with Lucifer Yellow, and by their relatively hyperpolarized membrane potentials and low input resistances (Table 1). An example of tracer spread in a retina perfused with control-Ringer's solution is illustrated in Figure 1. The tracers were introduced into a single astrocyte via a patch pipette and given $5 \mathrm{~min}$ to diffuse. At the end of this time, the live tissue was examined for Lucifer Yellow fluorescence. The dye was observed to have spread to several neighboring astrocytes, but not to Müller cells or neurons. Lucifer Yellow staining was still visible after fixation and processing of the tissue for the detection of neurobiotin (Fig. 1A), although the intensity of the labeling was reduced.

The spread of neurobiotin was far more extensive than was the spread of Lucifer Yellow. Neurobiotinfilled astrocytes and the endfeet of neurobiotin-filled Müller cells were observed over an extensive area on the vitreal surface (Fig. 1B). The profiles of labeled Müller cells could be fol lowed by focusing down into the retinal tissue using a conventional fluorescence microscope, but the labeling was much more clearly seen using a confocal microscope, which revealed welllabeled Müller cell somata in the inner nuclear layer (Fig. 1C). Labeled Müller cells could also be viewed in their entirety after three-dimensional computer reconstructions of optical sections (Fig. 1D,E).

Spread of Lucifer Yellow to two to ten neighboring astrocytes was observed in 98\% (39/40) of cells having membrane potentials of at least $-80 \mathrm{mV}\left(\mathrm{E}_{\mathrm{K}}\right.$ with the solutions used was $-101 \mathrm{mV}$ ). Lucifer Yell ow was never observed to spread to Müller cells or neurons. Neurobiotin was seen to label clusters of 13-88 astrocytes, covering areas 85-237 $\mu \mathrm{m}$ in diameter (see Fig. 7). Labeled Müller cells were confined to the central (approximately $100 \mu \mathrm{m}$ diameter) area of the region containing neurobiotin-filled astrocytes. Within this region, 118-288 Müller cells were labeled (see Fig. 7).

\section{Tracer spread from astrocytes to Müller cells is unidirectional}

To determine whether tracers could spread from an injected Müller cell to astrocytes or to other Müller cells, Müller cells were filled with tracers after patching onto their endfeet at the vitreal surface. The membrane potentials of Müller cells were generally more negative than those of astrocytes, and their input resistances were slightly less (Table 1). Twenty-five Müller cells, perfused with control-Ringer's solution, were filled with Lucifer Yellow; the dye was never seen to spread beyond the recorded cell. In 15 cases, neurobiotin was also included in the patch pipette. As with Lucifer Yellow, neurobiotin was never seen to spread beyond the injected cell (Fig. 2).

In a few experiments, astrocytes were injected with neurobiotin, but the retinas were not fixed until 20-40 minutes after the patch pipette was withdrawn. In these cases, clusters of neurobiotin-labeled Müller cells were observed after the retinas were processed, but there were no visibly labeled astrocytes (Fig. 3). These observations are consistent with unidirectional coupling between astrocytes and Müller cells: once the patch pipette was withdrawn from the injected astrocyte, there was no longer a continuous source of a high concentration of neurobiotin. With time, the neurobiotin diffused through the astrocyte syncytium until it reached concentrations that were no longer detectable. However, the neurobiotin that entered Müller cells did not diffuse out of these cells and remained at detectable concentrations.

\section{Effects of Uncoupling Agents on the Intercellular Spread of Lucifer Yellow and Neurobiotin}

Octanol and halothane, two agents that reduce dye coupling between cultured astrocytes, were tested for their ability to uncouple retinal glia in situ. 16-Doxylstearic acid (DSA), a compound shown to be effective in uncoupling cul tured cardiac myocytes (Burt, 1989), was al so tested. All the agents eliminated the visible spread of Lucifer Yellow between astrocytes and the visible spread of neurobiotin from astrocytes to Müller cells. However, only DSA in combination with octanol was effective in eliminating the visible spread of neurobiotin between astrocytes.

\section{Effects on the spread of L ucifer yellow between astrocytes}

Octanol $(500 \mu \mathrm{M})$ eliminated the visible spread of Lucifer Yellow between astrocytes in 95\% (63/66) of the cases studied (Fig. 4A,D); in the remaining 3 cases, it was not clear whether Lucifer Yell ow had spread to one other cell. In a few cases, the effects of lower doses of octanol were studied. The spread of Lucifer Yellow persisted in $100 \mu \mathrm{M}$ octanol but was eliminated in 200 


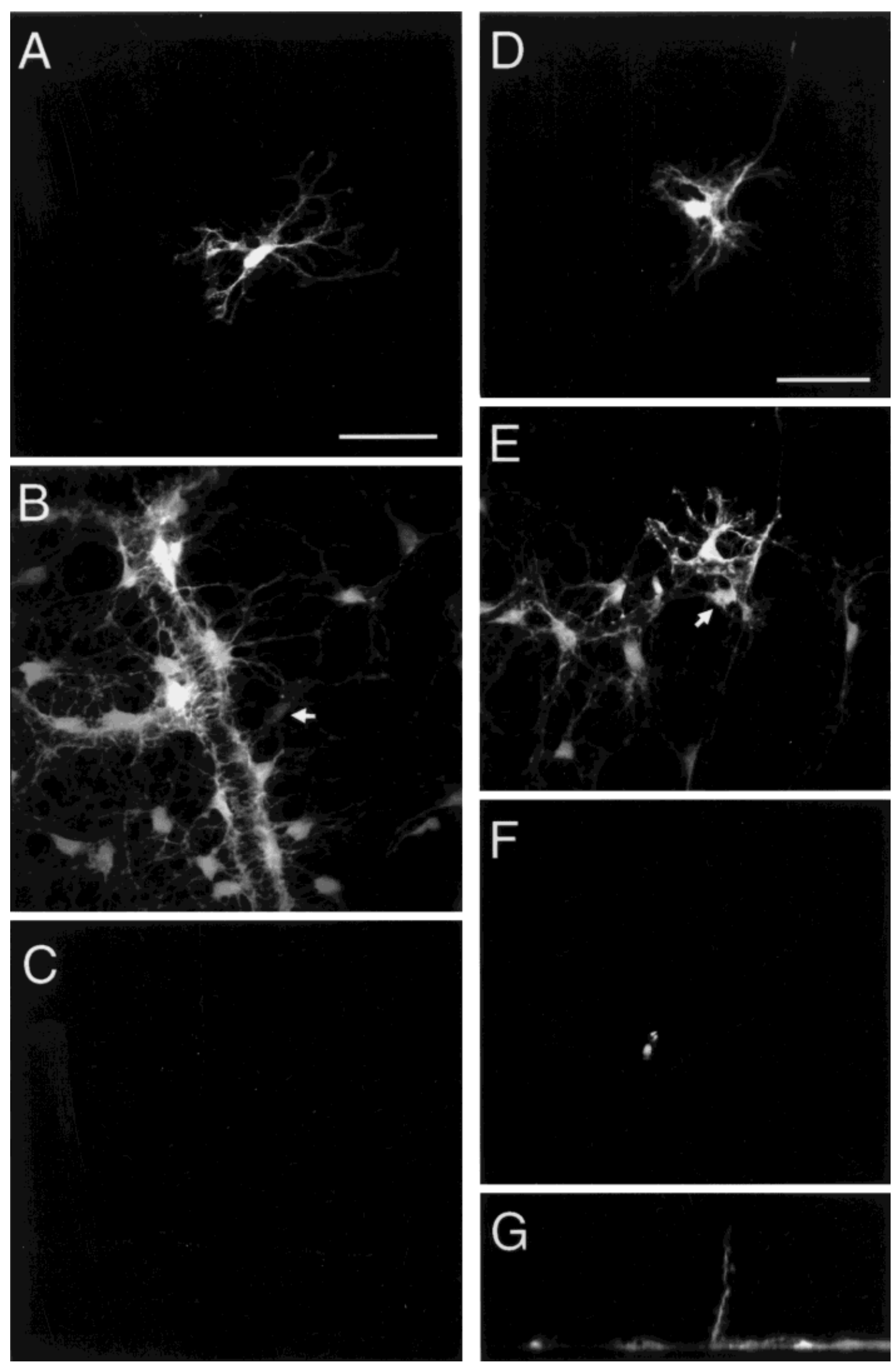

Fig. 4. 
$\mu \mathrm{M}$ octanol. To assess whether Lucifer Yellow spread from the injected cell at levels below our ability to detect it using fluorescence microscopy, astrocytes in 11 additional octanol $(500 \mu \mathrm{M})$ treated retinas were immunostained with an antibody directed against Lucifer Yellow. In all 11 cases, Lucifer Yellow was confined to the injected astrocyte. Halothane (4\%) also eliminated the visible spread of Lucifer Yellow between astrocytes in all 10 cases tested (Fig. 5A,B), as did DSA (50 and $100 \mu \mathrm{M}, 14$ cases total $)$, and DSA $(100 \mu \mathrm{M})$ in combination with octanol $(500 \mu \mathrm{M})(11$ cases; Fig. 6A,B). It was necessary to perfuse the retinas with DSA-containing solutions for at least $30 \mathrm{~min}$ to see a reliable effect on coupling.

\section{Effects on the spread of neurobiotin between astrocytes}

Octanol, at a concentration $(500 \mu \mathrm{M})$ that eliminated the visible spread of Lucifer Yell ow between astrocytes, did not reduce the visible spread of neurobiotin between astrocytes (Fig. 4B,E). In fact, astrocytes appeared to be more intensely labeled with neurobiotin in octanoltreated retinas compared with control retinas. The numbers of neurobiotin-label ed astrocytes and the areas they occupied were significantly larger in octanoltreated retinas compared with control retinas (Fig. 7). Theenhanced spread of neurobiotin between astrocytes may have resulted from octanol's effect of preventing the tracer from spreading into Müller cells. When the octanol concentration was increased to $1.0 \mathrm{mM}$, wholecell recordings could not be obtained, presumably because astrocytes were damaged.

Halothane (4\%) al so failed to el iminate the spread of neurobiotin between astrocytes (Figs. 5C,D, 7). Similarly, DSA (50 or $100 \mu \mathrm{M}$ ) failed to reduce the size of neurobi otin-labeled astrocyteclusters (Fig. 7). N o differences were observed between retinas treated with the

Fig. 4. The effects of octanol on dye coupling between retinal glial cells. Two experiments are shown, A-C representing one and D-G representing a second. In each case, an isolated retina was perfused with Ringer's solution containing $500 \mu \mathrm{M}$ octanol; a single astrocyte was recorded in the whole-cell configuration and filled with Lucifer Yellow and neurobiotin. A and D: Lucifer yellow labeling. Octanol eliminates the visible spread of Lucifer Yellow between astrocytes. B and E: Neurobiotin labeling. Octanol does not diminish the spread of neurobiotin between astrocytes, but it virtually eliminates the spread of neurobiotin from astrocytes to Müller cells. The same fields represented in $A$ and $D$ are shown in $B$ and $E$, respectively. The recorded cells are indicated by the arrows. (Attenuation of the Cy5 fluorescence was frequently seen in cells that were heavily labeled with Lucifer yellow, causing the neurobiotin-injected cell to appear less heavily labeled than its closest neighbors.) C and F: Neurobiotin labeling. Reconstructions of confocal images through the inner nuclear layers of the retinas illustrated in A and D, respectively. In one case (C), there are no Müller cell somata detectably labeled. In the second case $(F)$, the soma and process of a single Müller cell are labeled with neurobiotin. Because it was unusual to see any Müller cells labeled in octanol-treated retinas, it is possible that this Müller cell had been damaged by the patch pipette during its approach to the injected astrocyte. G: A 10- $\mu \mathrm{m}$-thick reconstructed slice perpendicular to the surface shows the single neurobiotin-filled Müller cell in $F$ and the labeled astrocytes at the vitreal surface (bottom). Scale bars $=50 \mu \mathrm{m}$; bar in A applies to A-C; bar in D applies to D-G. two doses of DSA, and data from the two groups were pooled for analysis. Only when DSA $(100 \mu \mathrm{M})$ was combined with octanol $(500 \mu \mathrm{M})$ did astrocytes appear to be effectively uncoupled, as assessed by the spread of neurobiotin (Figs. 6C,D, 7). In eight of ten retinas examined, neurobiotin was confined to the injected cell only; in the remaining two retinas, neurobiotin labeled clusters of six and eight astrocytes. The ethanol solvent used in the DSA experiments did not potentiate the effects of octanol; in a set of experiments in which ethanol (final concentration $0.2 \%$, equal to that in the DSA/octanol experiments) was added to octanol-Ringer's perfusate, neither the number of neurobiotinlabeled astrocytes $(63 \pm 14$, mean \pm SEM, $n=6)$ nor the area of retina containing labeled astrocytes $\left(0.14 \pm 0.03 \mathrm{~mm}^{2}, \mathrm{n}=6\right)$ differed si gnificantly from the values obtained in octanol-treated retinas.

\section{E ffects on the spread of neurobiotin between astrocytes and Müller cells}

Octanol $(500 \mu \mathrm{M})$ virtually el iminated the spread of neurobiotin from astrocytes to Müller cells (Figs. 4C,F,G, 7). A lower dose of octanol $(200 \mu \mathrm{M})$, which eliminated the interastrocytic spread of Lucifer Yellow, reduced, but did not eliminate, the number of neurobiotinlabel ed Müller cells in the few cases tested. Halothane (4\%; Figs. 5E ,F, 7), DSA (50 or $100 \mu \mathrm{M}$; Fig. 7), and DSA $(100 \mu \mathrm{M})$ in combination with octanol $(500 \mu \mathrm{M})$ (Figs. $6 C, D, 7)$ each eliminated the visible spread of neurobiotin from astrocytes to Müller cells.

\section{Effects on membrane properties}

We investigated the effects of uncoupling agents on membrane properties of both astrocytes and Müller cells. In astrocytes, neither the membrane potential nor the input resistance were significantly affected by octanol $(500 \mu \mathrm{M})$ or halothane $(4 \%)$. By contrast, DSA (50 or $100 \mu \mathrm{M}$ ) alone or in combination with octanol $(500 \mu \mathrm{M})$ significantly increased the input resistances of the recorded astrocytes, without affecting membrane potential. The ethanol solvent used in the DSA experiments did not itself affect these properties; in a set of experiments in which ethanol (final concentration $0.2 \%$ ) was added to control-Ringer's perfusate, neither the membrane potential $(-84 \pm 1 \mathrm{mV}, \mathrm{n}=12)$ nor the input resistance ( $26 \pm 2 M \Omega, n=12)$ differed significantly from control values.

In Müller cells, neither membrane potential nor input resistance were affected by octanol $(500 \mu \mathrm{M})$. Although membrane potential and input resistance of halothane-treated Müller cells were normal when the whole-cell configuration was initially achieved, membrane potential was unstable, and cel Is depol arized 2-3 min after the membrane was ruptured. In DSA (100 $\mu \mathrm{M})$ combined with octanol $(500 \mu \mathrm{M})$, the input resistances of Müller cells were quite variable, and there 

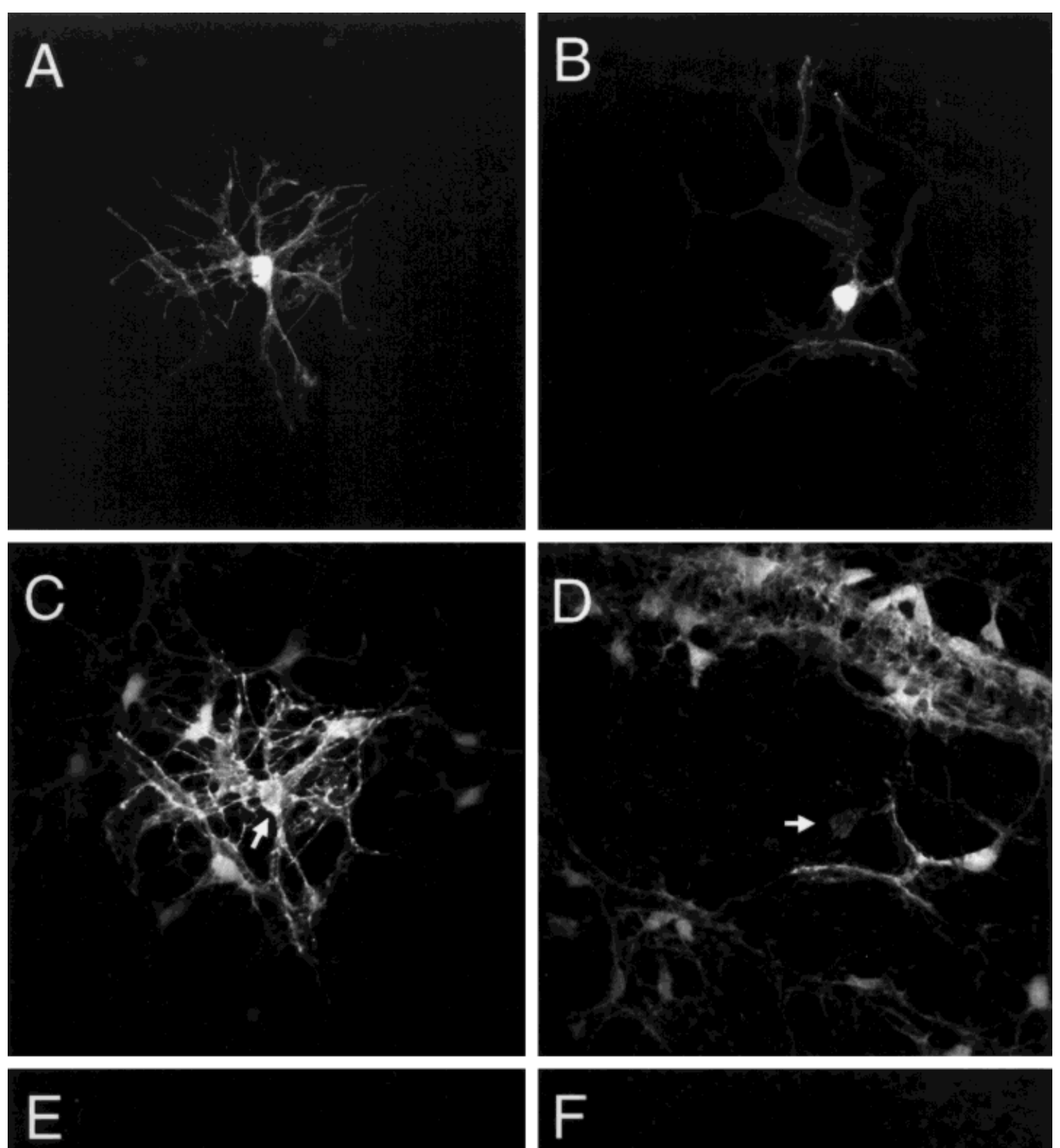

$F$

Fig. 5. 

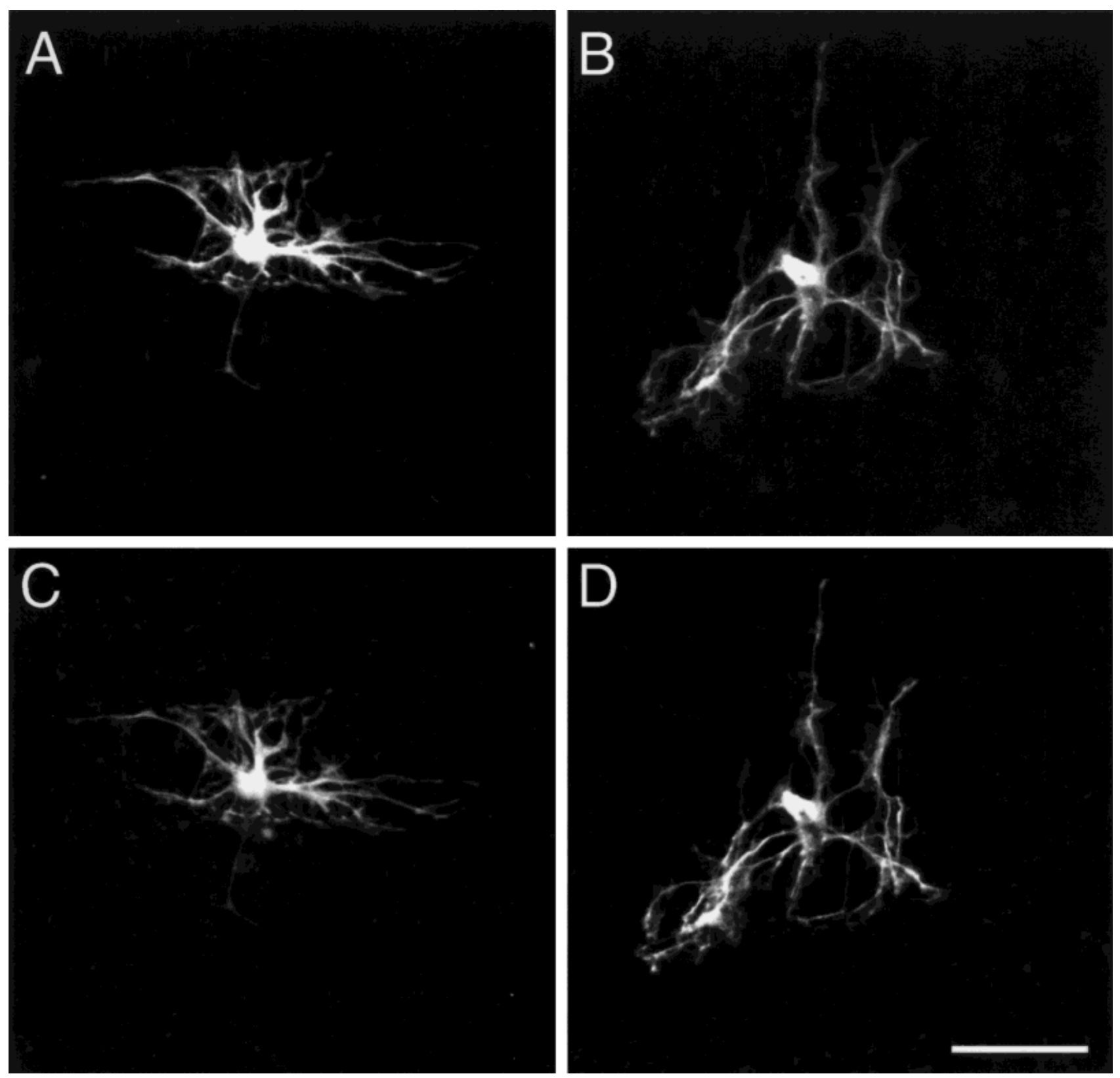

Fig. 6. DSA in combination with octanol eliminates tracer coupling between retinal glial cells. Examples from two experiments are shown. In each case, an isolated retina was perfused with Ringer's solution containing DSA $(100 \mu \mathrm{M})$ and octanol $(500 \mu \mathrm{M})$, and a single astrocyte was recorded in the whole-cell configuration and filled with Lucifer

Yellow and neurobiotin. Surface views of the retina. Both Lucifer Yellow (A) and (B) and neurobiotin (C) and (D) were confined to the recorded cell. The same fields represented in A and B are shown in C and $D$, respectively. Scale bar $=50 \mu \mathrm{m}$ and applies to all panels.

was not a statistically significant effect of these drugs. However, the membrane potentials of DSA/octanoltreated Müller cells were slightly depolarized compared with Müller cells perfused with control-Ringer's solution. These results are summarized in Table 1. Müller cells were not recorded in DSA alone. tin A and BiLucifer Yellow labeling. Hatothane spread of Lucifer Yellow between astrocytes. C and D: Neurobiotin labeling. Halothane does not eliminate the spread of neurobiotin between astrocytes, but it eliminates the spread of neurobiotin from astrocytes to Müller cells. The same fields represented in A and B are shown in C and D, respectively. The recorded cells are indicated by arrows. E and F: Neurobiotin labeling. Reconstructions of confocal images through the inner nuclear layers of the retinas illustrated in A and $B$, respectively. There are no Müller cell somata detectably labeled in either experiment. Scale bar $=50 \mu \mathrm{m}$ and applies to all panels.

\section{Glial cell labeling with neurobiotin is not due to uptake from the extracellular space}

To test whether the uptake of neurobiotin from the extracellular space accounted for any of our results, 

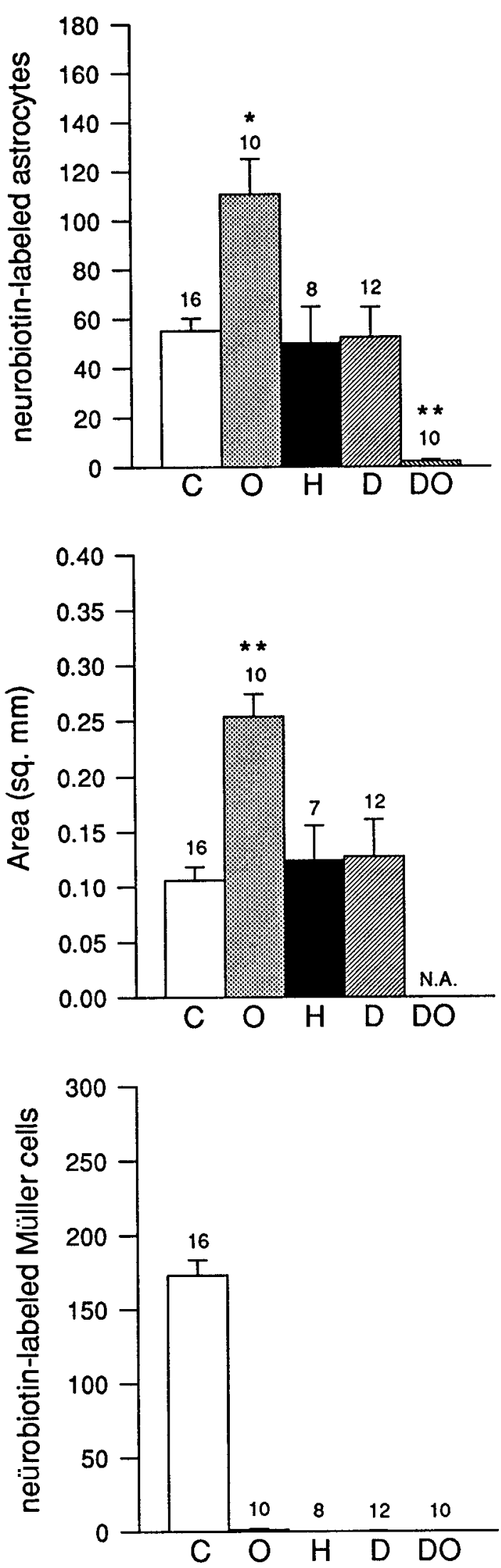

three retinas were incubated for $5 \mathrm{~min}$ at room temperature in oxygenated-Ringer's solution with $0.11,1.1$, or $11 \mathrm{mM}$ neurobiotin. The retinas were then fixed and incubated with streptavidin conjugated to Cy3. Specific labeling of glial cells was not observed in any of these retinas. At the two higher neurobiotin concentrations, there was detectable labeling in both plexiform layers.

\section{DISCUSSION}

The results presented here demonstrate that rat retinal astrocytes are dye-coupled in situ. Lucifer Yellow and neurobiotin introduced into single astrocytes with a patch pipette spread to neighboring astrocytes. In addition, neurobiotin, the smaller of the two tracers, was found to spread from astrocytes to Müller cells. Within the detection limits of our system, the transfer of neurobiotin from astrocytes to Müller cells was unidirectional. Neurobiotin injected into astrocytes spread into Müller cells, but when injected into Müller cells, the tracer did not spread into astrocytes. It should be noted that the astrocytes injected in this study were al ways located at a distance from retinal arteries and veins, and it is not certain whether injection of tracer into astrocyte somata closely apposed to these blood vessels would result in the same pattern of coupling.

Our results in the rat retina are similar to findings in the rabbit retina (Robinson et al., 1993), although the two species differ in some respects. In the rabbit, Lucifer Yellow injected into astrocytes spread to neighboring Müller cells in more than $10 \%$ of the cases, whereas spread of Lucifer Yellow from astrocyte to Müller cell was never observed in the rat. In addition, the spread of neurobiotin from astrocytes to Müller cells in the rat retina was moreextensivethan was thespread of biocytin from astrocytes to Müller cells in the rabbit retina. Whether these dissimilarities reflect true species differences or methodological differences is not dear.

\section{Rectifying Gap J unctions}

Chemically rectifying gap junctions (i.e., gap junctions in which some molecules pass more readily in one

Fig. 7. Summary of the effects of uncoupling agents on neurobiotin transfer between retinal glial cells. Top: The number of neurobiotinlabeled astrocytes counted in each retina after the introduction of neurobiotin into a single astrocyte. Middle: The area over which neurobiotin-labeled astrocytes were found after the introduction of neurobiotin into a single astrocyte. Bottom: The number of neurobiotin-labeled Müller cell somata counted in a $100-\mu \mathrm{m}$-diameter circle within the inner nuclear layer after the introduction of neurobiotin into a single astrocyte. In each graph, the mean and SEM are illustrated for retinas perfused with control-Ringer's (C), Ringer's with $500 \mu \mathrm{M}$ octanol $(\mathrm{O})$, Ringer's bubbled with $\mathrm{O}_{2}$ with $4 \%$ halothane $(\mathrm{H})$, Ringer's with 50 or $100 \mu \mathrm{M}$ DSA (D), and Ringer's with $100 \mu \mathrm{M}$ DSA plus $500 \mu \mathrm{M}$ octanol (DO). Numbers above the bars show the number of retinas included in each sample. N.A., not applicable; measurements not made in DSA/octanol-treated retinas, whereneurobiotin did not spread from the injected cell. Value is statistically different from that found in retinas perfused with control-Ringer's, Student's t-test: *, $\mathrm{P}<0.001 ; * *, \mathrm{P}<10^{-6}$. For the bottom graph, all groups are statistically different from control at $\mathrm{P}<10^{-6}$. 
direction than in the other) were first demonstrated between heterotypic cell pairs in vitro (Flagg-Newton and Loewenstein, 1980). Chemical rectification between cells in situ has thus far been observed only in the retina, first between rabbit retinal glial cells (Robinson et al., 1993), and now between rat retinal glia.

Although the gap junctional pore is relatively nonselective compared with other types of membrane channels, junctional channels do exhibit some degree of selective permeability, based on the size, charge, and shape of the permeants (Elfgang et al., 1995; Loewenstein, 1981). In order for chemical rectification to occur, the hemi-channels contributed by each cell must have different permselectivities, and there must exist "an asymmetric free-energy barrier for permeant movement in the channel that is higher in one direction than in the other" (Loewenstein, 1981). Asymmetry between hemi-channels could arise if they are made from different connexins, the proteins that form gap junctions.

\section{Sensitivity to Uncoupling Agents}

Astrocyte-to-M üller cell coupling was more sensitive to gap-junction blockers than was astrocyte-to-astrocyte coupling. The spread of neurobiotin between astrocytes persisted in octanol, halothane, and DSA, all of which eliminated the spread of tracer from astrocytes to Müller cells. It is perhaps not surprising that the gap junctions between astrocytes and Müller cells have different sensitivities to uncoupling agents than do the gap junctions between astrocytes. The asymmetry of the astrocyte-to-Müller cell junction implies that the hemi-channels contributed by each cell type are formed from different connexins. Although the connexins expressed by Müller cells and by retinal astrocytes have yet to be identified, it has been shown that connexins can vary in their sensitivity to uncoupling agents (Moreno et al., 1991; Rup et al., 1993).

The extent to which interastrocytic coupling persisted in the presence of the uncoupling agents was surprising. Concentrations of all three agents that el iminated the visible spread of Lucifer Yellow between astrocytes did not visibly diminish the spread of neurobiotin. However, some reduction in the transfer of neurobiotin from the injected cell might not necessarily be revealed by our data. Our measures of tracer spread are meant to provide a qualitative assessment of the efficacy of the uncoupling agents tested, but they do not provide quantitative information about the amount of neurobiotin in a label ed cell. The simplest explanation for our results is that interastrocytic coupling is mediated by a single type of junctional channel that is permeable to both Lucifer Yellow and neurobiotin. The uncoupling agents either: 1) reduce the flux of Lucifer Yellow through this channel more than they reduce the flux of the smaller neurobiotin; or 2) equally attenuate the spread of the two tracers, but we are less able to detect reductions in the spread of neurobiotin. At least for octanol, the latter explanation seems unlikely. In octanol-treated retinas, the number of detectably labeled astrocytes was greater than in control retinas, whereas there was no detectable spread of Lucifer Yellow, using either fluorescence microscopy or immunoperoxidase techniques to visual ize the dye.

Regardless of the underlying mechanisms, our results confirm that monitoring the visible spread of Lucifer Yellow can be inadequate for assessing the presence of junctional coupling between cells (Peinado et al., 1993; Ransom and Kettenmann, 1990; Vaney, 1994).

\section{Increased Input Resistance in DSA-Treated Astrocytes}

DSA, alone or in combination with octanol, significantly increased the input resistance of retinal astrocytes. The average input resistance of cells treated with DSA/octanol, which appeared to eliminate tracer coupling between astrocytes, was approximately double the average input resistance of astrocytes in controlRinger's solution. We do not know whether this change in resistance solely reflects the cl osure of gap junctional channels or whether non-junctional conductances were also affected. In the experiments with DSA alone, the changes in input resistance were not paralleled by changes in the number of labeled astrocytes. This observation does not absolutely imply that there are non-junctional effects, however, since these measures of tracer spread do not necessarily bear a linear relationship to junctional conductance.

\section{F unctions of Glial Cell Coupling}

Although the functions of the coupling between retinal glia have yet to be determined, interastrocytic coupling is thought to have a role in $\mathrm{K}^{+}$spatial buffering (Orkand et al., 1966), in the propagation of intercelIular $\mathrm{Ca}^{2+}$ waves (Charles et al., 1992; Enkvist and McCarthy, 1992; F inkbeiner, 1992; Nedergaard, 1994), and in the coordination of metabolic activity between glial cells (Tabernero et al., 1996). Under the spatial buffer hypothesis, $\mathrm{K}^{+}$released from active neurons enters neighboring glial cells and is redistributed by current flow through the glial syncytium. It seems unlikely that coupling between retinal astrocytes has an important role in $\mathrm{K}^{+}$buffering, however, since it would be more efficient for astrocytes to release $\mathrm{K}^{+}$ directly into the vitreous humor, only a few micrometers away. It has recently been reported that both astrocytes and Müller cells participatein $\mathrm{Ca}^{2+}$ waves in the retina (Newman and Zahs, 1997), and the gap junctions between them might provide a route for propagation of these waves.

It remains to be determined to what degree the astrocyte-to-Müller cell coupling observed in the isolated retina reflects the situation in vivo. Although gap junctions on mammalian Müller cells have been found 
in a few instances (Burns and Tyler, 1990; LaVail et al., 1974; Reale et al., 1978), they are not commonly reported in ultrastructural studies of the retina (Bussow, 1980; Hollander et al., 1991; Uga and Smelser, 1973). It is conceivable that preparation of the isolated retina induces a change in the pattern or extent of coupling between retinal glial cells. Cells in our study were filled with tracers 1-6 $\mathrm{h}$ after isol ating the retinas, and connexin-43 immunoreactivity has been shown to increase in astrocytes of the facial nucleus within an hour after transection of the facial nerve (Rohlmann et al., 1994).

It is intriguing to speculate that the gap junctions between astrocytes and between astrocytes and Müller cells can be modulated independently by physiological signals. Glial cell coupling has been shown to increase with nerve activity (Marrero and Orkand, 1996) and to decrease after activation of protein kinase C (Enkvist and McCarthy, 1992). I ndependent modulation of homotypic and heterotypic coupling has recently been demonstrated in the retina, where the gap junctions between All amacrine cells and between AlI amacrine cells and ON-bipolar cells can be differentially modulated by agents that increase levels of intracellular CAMP and cGMP, respectively (Mills and Massey, 1995).

Gap junctions between astrocytes are ubiquitous, and gap junctions between astrocytes and other glial types are not uncommon. Yet the physiological roles of junctional coupling are largely speculative. The retina may provide a useful model for studying the functions and regulation of gap junctional communication between neuroglial cells.

\section{ACKNOWLEDGMENTS}

We thank Paul Ceelen for technical assistance and Robert Wolfe for assistance with confocal microscopy.

\section{REFERENCES}

Binmoller, F.-J . and Müller, C.M. (1992) Postnatal development of dye-coupling among astrocytes in rat visual cortex. Glia, 6:127-137.

Burns, M.S. and Tyler, N.K. (1990) Interglial cell gap junctions increase in urethane-induced photoreceptor degeneration in rats. Invest. Ophthalmol. Vis. Sci., 31:1690-1701.

Burt, J.M. (1989) Uncoupling of cardiac cells by doxyl stearic acids: Specificity and mechanism of action. Am. J. Physiol., 256:C913C924.

Bussow, H. (1980) The astrocytes in the retina and optic nerve head of mammals: A special glia for the ganglion cell axons. Cell TissueRes., 206:367-378.

Charles, A.C., Naus, C.C.G., Zhu, D., Kidder, G.M., Dirksen, E.R., and Sanderson, M.J. (1992) Intercellular calcium signaling via gap junctions in glioma cells. J . Cell Biol., 118:195-201.

Elfgang, C., Eckert, R., Lichtenberg-F rate, H., and Butterweck, A. (1995) Specific permeability and selective formation of gap junction channels in connexin-transfected HeLa cells. J . Cell Biol., 129:805817.

Enkvist, K.M.O. and McCarthy, K.D. (1992) Activation of protein kinase $C$ blocks astroglial gap junction communication and inhibits the spread of calcium waves. J . Neurochem., 59:519-526.
Enkvist, K.M.O. and McCarthy, K.D. (1994) Astroglial gap junction communication is increased by treatment with either glutamate or high $\mathrm{K}^{+}$concentration. J . Neurochem., 62:489-495.

Finkbeiner, S. (1992) Calcium waves in astrocytes-filling in the gaps. Neuron, 8:1101-1108.

Flagg-Newton, J.L. and Loewenstein, W.R. (1980) Asymmetrically permeable membrane channels in cell junctions. Science, 207:771773.

Hollander, H., Makarov, F., Dreher, Z., Driel, D.V., Chan-Ling, T., and Stone, J . (1991) Structure of the macroglia of the retina: Sharing and division of labour between astrocytes and Müller cells. J . Comp. Neurol., 313:587-603.

LaVail, M.M., Sidman, M., Rausin, R., and Sidman, R.L. (1974) Discrimination of light intensity by rats with inherited retinal degeneration. Vision Res., 14:693-702.

Loewenstein, W.R. (1981) J unctional intercellular communication: The cell-to-cell membrane channel. Physiol. Rev., 61:829-913.

Marrero, H. and Orkand, R.K. (1996) Nerve impulses increase glial intercellular permeability. Glia, 16:285-289.

Massa, P.T. and Mugnaini, E. (1982) Cell junctions and intramembrane particles of astrocytes and oligodendrocytes: A freeze-fracture study. Neuroscience, 7:523-538.

Massa, P.T. and Mugnaini, E. (1985) Cell-cell junctional interactions and characteristic plasma membrane features of cultured rat glial cells. Neuroscience, 14:695-709.

Mills, S.L. and Massey, S.C. (1995) Differential properties of two gap junctional pathways made by All amacrine cells. Nature, 377:734737.

Moreno, A.P., Eghbali, B., and Spray, D.C. (1991) Connexin32 gap junction channels in stably transfected cells: Unitary conductance. Biophys. J ., 60:1254-1266.

Naus, C.C.G., Hearn, S., Zhu, D., Nicholson, B.J ., and Shivers, R.R. (1993) Ultrastructural analysis of gap junctions in C6 glioma cells transfected with connexin43 cDNA. Exp. Cell Res., 206:72-84.

Nedergaard, M. (1994) Direct signaling from astrocytes to neurons in cultures of mammalian brain cells. Science, 263:1768-1771.

Newman, E.A. and Zahs, K.R. (1997) Calcium waves in retinal glial cells. Science, 275:844-47.

Orkand, R.K., Nicholls, J.G., and Kuffler, S.W. (1966) Effect of nerve impulses on the membrane potential of glial cells in the central nervous system of amphi bia. J. Neurophysiol., 29:788-806.

Peinado, A., Yuste, R., and Katz, L.C. (1993) Extensive dye coupling between rat neocortical neurons during the period of circuit formation. Neuron, 10:103-114.

Ransom, B.R. and Kettenmann, H. (1990) Electrical coupling, without dye coupling, between mammalian astrocytes and oligodendrocytes in cell culture. Glia, 3:258-266.

Reale, E., Luciano, L., and Spitznas, M. (1978) Communicating junctions of the human sensory retina. Graefes. Arch. Klin. Exp. Ophthalmol ., 208:77-92.

Robinson, S.R., Hampson, E.C.G.M., Munro, M.N., and Vaney, D.I. (1993) Unidirectional coupling of gap junctions between neuroglia. Science, 262:1072-1074.

Rohlmann, A., Laskawi, R., Hofer, A., Dermietzel, R., and Wolff, J .R. (1994) Astrocytes as rapid sensors of peripheral axotomy in the facial nucleus of rats. Neuroreport, 5:409-412.

Rup, D.M., Veenstra, R.D., Wang, H.-Z., Brink, P.R., and Beyer, E.C. (1993) Chick connexin-56, a novel lens gap junction protein: Molecular cloning and functional expression. J. Biol. Chem., 268:706-712.

Tabernero, A., Giaume, C., and Medina, J. (1996) Endothelin-1 regulates glucose utilization in cultured astrocytes by controlling intercellular communication through gap junctions. Glia, 16:187195.

Tani, E., Nishiura, M., and Higashi, M. (1973) Freeze-fracture studies of gap junctions of normal and neoplastic astrocytes. Acta Neuropathol., 26:127-138.

Uga, S. and Smelser, G.K. (1973) Comparative study of the fine structure of retinal Müller cells in various vertebrates. Invest. Ophthalmol., 12:434-448.

Vaney, D.I. (1994) Pattern of neuronal coupling in the retina. Prog. Retinal EyeRes., 13:301-355.

Venance, L., Cordier, J., Monge, M., Zalc, B., Glowinski, J ., and Giaume, C. (1995) Homotypic and heterotypic coupling mediated by gap junctions during glial cell differentiation in vitro. Eur. J. Neurosci. 7:451-461.

Zhu, D., Caveny, S., Kidder, G.M., and Naus, C.C. (1991) Transfection of C6 glioma cells with connexin43 cDNA: Analysis of expression, intercellular coupling, cell proliferation. Proc. Natl. Acad. Sci., U.S.A., 88:1883-1887. 